\title{
Inequality estimates for the boundedness of multilinear singular and fractional integral operators
}

\author{
Xiaosha Zhou, Chuangxia Huang ${ }^{*}$, Haijun Hu and Li Liu
}

\section{"Correspondence:}

cxiahuang@126.com

Department of Mathematics,

Changsha University of Science and

Technology, Changsha, 410077, P.R. China

\begin{abstract}
In this paper, the inequality of boundedness for the multilinear fractional singular integral operators associated to the weighted Lipschitz functions is estimated. The operators include the Calderón-Zygmund singular integral operator and the fractional integral operator.

MSC: 42B20; 42B25

Keywords: multilinear operators; Calderón-Zygmund singular integral operator; fractional integral operator; weighted Lipschitz space; Triebel-Lizorkin space
\end{abstract}

\section{Introduction}

As the development of the singular integral operators, their commutators and multilinear operators have been well studied (see [1-5]). In [1-3, 5-7], the authors proved that the commutators and multilinear operators generated by the singular integral operators and $B M O$ functions are bounded on $L^{p}\left(R^{n}\right)$ for $1<p<\infty$. Chanillo (see [8]) proved a similar result when singular integral operators are replaced by the fractional integral operators. In [9-12], the boundedness for the commutators and multilinear operators generated by the singular integral operators and Lipschitz functions on $L^{p}\left(R^{n}\right)(1<p<\infty)$ and TriebelLizorkin spaces are obtained. In $[13,14]$, the weighted boundedness for the commutators generated by the singular integral operators and $B M O$ or Lipschitz functions on $L^{p}\left(R^{n}\right)$ $(1<p<\infty)$ spaces are obtained. The purpose of this paper is to study the weighted boundedness for some multilinear operators associated to the fractional singular integral operators and the weighted Lipschitz functions. As application, the weighted boundedness for the multilinear operators associated to the Calderón-Zygmund singular integral operator and the fractional integral operator is obtained.

\section{Notations and theorems}

In this paper, we are going to consider some multilinear operators as follows.

Let $m_{j}$ be positive integers $(j=1, \ldots, k), m_{1}+\cdots+m_{k}=m$, and let $b_{j}$ be locally integrable functions on $R^{n}(j=1, \ldots, k)$. Set

$$
R_{m_{j}+1}\left(b_{j} ; x, y\right)=b_{j}(x)-\sum_{|\alpha| \leq m_{j}} \frac{1}{\alpha !} D^{\alpha} b_{j}(y)(x-y)^{\alpha} .
$$

@ 2013 Zhou et al.; licensee Springer. This is an Open Access article distributed under the terms of the Creative Commons Attribution License (http://creativecommons.org/licenses/by/2.0), which permits unrestricted use, distribution, and reproduction in any medium, provided the original work is properly cited. 
Definition 1 Let $T: S \rightarrow S^{\prime}$ be a linear operator, and there exists a locally integrable function $K(x, y)$ on $R^{n} \times R^{n}$ such that

$$
T(f)(x)=\int_{R^{n}} K(x, y) f(y) d y
$$

for every bounded and compactly supported function $f$, where $K$ satisfies: for fixed $0 \leq$ $\delta<n$ and $0<\varepsilon \leq 1$,

$$
|K(x, y)| \leq C|x-y|^{-n+\delta},
$$

and

$$
|K(y, x)-K(z, x)| \leq C|y-z|^{\varepsilon}|x-z|^{-n+\delta-\varepsilon} \quad \text { if } 2|y-z| \leq|x-z| .
$$

Given bounded and compactly supported functions $f$ defined on $R^{n}$, the multilinear operator associated to $T$ is defined by

$$
T^{b}(f)(x)=\int_{R^{n}} \frac{\prod_{j=1}^{k} R_{m_{j}+1}\left(b_{j} ; x, y\right)}{|x-y|^{m-k}} K(x, y) f(y) d y .
$$

Note that when $m=0, T^{b}$ is just a multilinear commutator of $T$ and $b$ (see $[15,16]$ ), while when $m>0$, it is non-trivial generalization of the commutators; when $\eta=0, T^{b}$ is just a multilinear commutator of the singular integral operator, when $0 \leq \eta<n, T^{b}$ is just a multilinear commutator of the fractional integral operator. It is well known that multilinear operators are of great interest in harmonic analysis and have been widely studied by many authors (see $[1-4,6,7,9,10,15,16])$. The purpose of this paper is to study the weighted boundedness properties for the multilinear operator.

Throughout this paper, $Q$ will denote a cube of $R^{n}$ with sides parallel to the axes. For a cube $Q$ and a locally integrable function $f$, let $f(Q)=\int_{Q} f(x) d x, f_{Q}=|Q|^{-1} \int_{Q} f(x) d x$ and

$$
f^{\#}(x)=\sup _{Q \ni x} \frac{1}{|Q|} \int_{Q}\left|f(y)-f_{Q}\right| d y .
$$

It is well known that (see $[17,18])$

$$
f^{\#}(x) \approx \sup _{Q \ni x} \inf _{C \in C} \frac{1}{|Q|} \int_{Q}|f(y)-c| d y .
$$

For $1 \leq p<\infty$ and $0 \leq \eta<n$, let

$$
M_{\eta, p}(f)(x)=\sup _{Q \ni x}\left(\frac{1}{|Q|^{1-p \eta / n}} \int_{Q}|f(y)|^{p} d y\right)^{1 / p},
$$

which is the Hardy-Littlewood maximal function when $p=1$ and $\eta=0$.

The $A_{p}$ weight is defined by (see [17])

$$
\begin{aligned}
& A_{p}=\left\{w: \sup _{Q}\left(\frac{1}{|Q|} \int_{Q} w(x) d x\right)\left(\frac{1}{|Q|} \int_{Q} w(x)^{-1 /(p-1)} d x\right)^{p-1}<\infty\right\}, \quad 1<p<\infty, \\
& A_{1}=\{w>0: M(w)(x) \leq C w(x), \text { a.e. }\}
\end{aligned}
$$


and $A_{\infty}=\bigcup_{p \geq 1} A_{p}$. We know, for $w \in A_{1}, w$ satisfies the double condition, that is, for any cube $Q$,

$$
w(2 Q) \leq C w(Q) .
$$

The $A(p, q)$ weight is defined by (see [19])

$$
\begin{aligned}
A(p, q)= & \left\{w>0: \sup _{Q}\left(\frac{1}{|Q|} \int_{Q} w(x)^{q} d x\right)^{1 / q}\left(\frac{1}{|Q|} \int_{Q} w(x)^{-p /(p-1)} d x\right)^{(p-1) / p}<\infty\right\}, \\
& 1<p, q<\infty .
\end{aligned}
$$

Given a weight function $w$. For $1<p<\infty$, the weighted Lebesgue space $L^{p}(w)$ is the space of functions $f$ such that

$$
\|f\|_{L^{p}(w)}=\left(\int_{R^{n}}|f(x)|^{p} w(x) d x\right)^{1 / p}<\infty
$$

For $\beta>0$ and $p>1$, let $\dot{F}_{p}^{\beta, \infty}(w)$ be the weighted homogeneous Triebel-Lizorkin space. For $0<\beta<1$, the weighted Lipschitz space $\operatorname{Lip}_{\beta}(w)$ is the space of functions $f$ such that

$$
\|f\|_{\operatorname{Lip}_{\beta}(w)}=\sup _{Q} \frac{1}{w(Q)^{1+\beta / n}} \int_{Q}\left|f(y)-f_{Q}\right| d y<\infty .
$$

We shall prove the following theorems in Section 3.

Theorem 1 Suppose that $T^{b}$ is the multilinear operator as Definition 1 such that $T$ is bounded from $L^{u}\left(R^{n}\right)$ to $L^{v}\left(R^{n}\right)$ for any $0 \leq \eta<n, 1<u<n / \eta$ and $1 / u-1 / v=\eta / n$. Let $0<\beta \leq 1, \beta+\delta<n, w \in A_{1}$ and $D^{\alpha} b_{j} \in \operatorname{Lip}_{\beta}(w)$ for all $\alpha$ with $|\alpha|=m_{j}$ and $j=1, \ldots, k$. Then $T^{b}$ is bounded from $L^{p}(w)$ to $L^{q}\left(w^{1-q(k-(\delta+k) / n)}\right)$ for any $1<p<n /(\delta+k+k \beta)$ and $1 / p-1 / q=(\delta+k+k \beta) / n$.

Theorem 2 Suppose that $T^{b}$ is the multilinear operator as Definition 1 such that $T$ is bounded from $L^{u}\left(R^{n}\right)$ to $L^{v}\left(R^{n}\right)$ for any $0 \leq \eta<n, 1<u<n / \eta$ and $1 / u-1 / v=\eta / n$. Let $0<\beta<$ $\min (1 / k, \varepsilon / k), \beta+\delta<n, w \in A_{1}$ and $D^{\alpha} b_{j} \in \operatorname{Lip}_{\beta}(w)$ for all $\alpha$ with $|\alpha|=m_{j}$ and $j=1, \ldots, k$. Then $T^{b}$ is bounded from $L^{p}(w)$ to $\dot{F}_{q}^{k \beta, \infty}\left(w^{1-q(k-(\delta+k-k \beta) / n)}\right)$ for any $1<p<n /(\delta+k)$ and $1 / p-1 / q=(\delta+k) / n$.

\section{Proofs of theorems}

We begin with some preliminary lemmas.

Lemma 1 (see [1]) Let b be a function on $R^{n}$ and $D^{\alpha} b \in L^{q}\left(R^{n}\right)$ for $|\alpha|=m$ and some $q>n$. Then

$$
\left|R_{m}(b ; x, y)\right| \leq C|x-y|^{m} \sum_{|\alpha|=m}\left(\frac{1}{|\tilde{Q}(x, y)|} \int_{\tilde{Q}(x, y)}\left|D^{\alpha} b(z)\right|^{q} d z\right)^{1 / q},
$$

where $\tilde{Q}(x, y)$ is the cube centered at $x$ and having side length $5 \sqrt{n}|x-y|$. 
Lemma 2 (see $[11,12]$ ) For $0<\beta<1,1<p<\infty$ and $w \in A_{\infty}$, we have

$$
\begin{aligned}
\|f\|_{\dot{F}_{p}^{\beta, \infty}(w)} & \approx\left\|\sup _{Q \ni \cdot} \frac{1}{|Q|^{1+\beta / n}} \int_{Q}\left|f(x)-f_{Q}\right| d x\right\|_{L^{p}(w)} \\
& \approx\left\|\sup _{Q \ni \cdot c} \frac{1}{|Q|^{1+\beta / n}} \int_{Q}|f(x)-c| d x\right\|_{L^{p}(w)} .
\end{aligned}
$$

Lemma 3 (see [8]) Suppose that $1 \leq s<p<n / \eta, 1 / q=1 / p-\eta / n$ and $w \in A(p, q)$. Then

$$
\left\|M_{\eta, s}(f)\right\|_{L^{q}\left(w^{q}\right)} \leq C\|f\|_{L^{p}\left(w^{p}\right)}
$$

Lemma 4 (see $[17,20]$ ) For $0<\beta<1,1 \leq p \leq \infty$ and $w \in A_{1}$, we have

$$
\|b\|_{\operatorname{Lip}_{\beta}(w)} \approx \sup _{Q} \frac{1}{w(Q)^{\beta / n}}\left(\frac{1}{w(Q)} \int_{Q}\left|b(x)-b_{Q}\right|^{p} w(y)^{1-p} d x\right)^{1 / p} .
$$

Lemma 5 (see [20]) For any cube $Q, b \in \operatorname{Lip}_{\beta}(w), 0<\beta<1$ and $w \in A_{1}$, we have

$$
\sup _{x \in Q}\left|b(x)-b_{Q}\right| \leq C\|b\|_{\operatorname{Lip}_{\beta}(w)} w(Q)^{1+\beta / n}|Q|^{-1} .
$$

To prove the theorems, we need the following lemmas.

Key Lemma 1 Suppose that $T^{b}$ is the multilinear operator as Definition 1 such that $T$ is bounded from $L^{u}\left(R^{n}\right)$ to $L^{v}\left(R^{n}\right)$ for any $0 \leq \eta<n, 1<u<n / \eta$ and $1 / u-1 / v=\eta / n$. Let $0<\beta \leq 1, \beta+\delta<n, w \in A_{1}$ and $D^{\alpha} b_{j} \in \operatorname{Lip}_{\beta}(w)$ for all $\alpha$ with $|\alpha|=m_{j}$ and $j=1, \ldots, k$. Then there exists a constant $C_{0}$ such that for every $f \in C_{0}^{\infty}\left(R^{n}\right), 1<s<n / \delta$ and $\tilde{x} \in R^{n}$,

$$
\begin{aligned}
& \sup _{Q \ni \tilde{x}} \frac{1}{|Q|} \int_{Q}\left|T^{b}(f)(x)-C_{0}\right| d x \\
& \quad \leq C \prod_{j=1}^{k}\left(\sum_{\left|\alpha_{j}\right|=m_{j}}\left\|D^{\alpha_{j}} b_{j}\right\|_{\operatorname{Lip}_{\beta}(w)}\right) w(\tilde{x})^{k+k \beta / n} M_{\delta+k+k \beta, s}(f)(\tilde{x}) .
\end{aligned}
$$

Key Lemma 2 Suppose that $T^{b}$ is the multilinear operator as Definition 1 such that $T$ is bounded from $L^{u}\left(R^{n}\right)$ to $L^{v}\left(R^{n}\right)$ for any $0 \leq \eta<n, 1<u<n / \eta$ and $1 / u-1 / v=\eta / n$. Let $0<\beta<\min (1 / k, \varepsilon / k), \beta+\delta<n, w \in A_{1}$ and $D^{\alpha} b_{j} \in \operatorname{Lip}_{\beta}(w)$ for all $\alpha$ with $|\alpha|=m_{j}$ and $j=1, \ldots, k$. Then there exists a constant $C_{0}$ such that for every $f \in C_{0}^{\infty}\left(R^{n}\right), 1<s<n / \delta$ and $\tilde{x} \in R^{n}$

$$
\begin{aligned}
& \sup _{Q \ni \tilde{x}} \frac{1}{|Q|^{1+k \beta / n}} \int_{Q}\left|T^{b}(f)(x)-C_{0}\right| d x \\
& \quad \leq C \prod_{j=1}^{k}\left(\sum_{\left|\alpha_{j}\right|=m_{j}}\left\|D^{\alpha_{j}} b_{j}\right\|_{\operatorname{Lip}_{\beta}(w)}\right) w(\tilde{x})^{k+k \beta / n} M_{\delta+k, s}(f)(\tilde{x}) .
\end{aligned}
$$

Proof of Key Lemma 1 Without loss of generality, we may assume $k=2$. Fix a cube $Q=Q\left(x_{0}, d\right)$ with $Q \ni \tilde{x}$. Let $\tilde{Q}=5 \sqrt{n} Q$ and $\tilde{b}_{j}(x)=b_{j}(x)-\sum_{|\alpha|=m} \frac{1}{\alpha !}\left(D^{\alpha} b_{j}\right)_{\tilde{Q}^{x^{\alpha}}}$, then 
$R_{m+1}\left(b_{j} ; x, y\right)=R_{m+1}\left(\tilde{b}_{j} ; x, y\right)$ and $D^{\alpha} \tilde{b}_{j}=D^{\alpha} b_{j}-\left(D^{\alpha} b_{j}\right)_{\tilde{Q}}$ for $|\alpha|=m_{j}$. We split $f=f \chi_{\tilde{Q}}+$ $f \chi_{R^{n} \backslash \tilde{Q}}=f_{1}+f_{2}$. Write

$$
\begin{aligned}
T^{b}(f)(x)= & \int_{R^{n}} \frac{\prod_{j=1}^{2} R_{m_{j}}\left(\tilde{b}_{j} ; x, y\right)}{|x-y|^{m-2}} K(x, y) f_{1}(y) d y \\
& -\sum_{\left|\alpha_{1}\right|=m_{1}} \frac{1}{\alpha_{1} !} \int_{R^{n}} \frac{R_{m_{2}}\left(\tilde{b}_{2} ; x, y\right)(x-y)^{\alpha_{1}}}{|x-y|^{m-2}} D^{\alpha_{1}} \tilde{b}_{1}(y) K(x, y) f_{1}(y) d y \\
& -\sum_{\left|\alpha_{2}\right|=m_{2}} \frac{1}{\alpha_{2} !} \int_{R^{n}} \frac{R_{m_{1}}\left(\tilde{b}_{1} ; x, y\right)(x-y)^{\alpha_{2}}}{|x-y|^{m-2}} D^{\alpha_{2}} \tilde{b}_{2}(y) K(x, y) f_{1}(y) d y \\
& +\sum_{\left|\alpha_{1}\right|=m_{1},\left|\alpha_{2}\right|=m_{2}} \frac{1}{\alpha_{1} ! \alpha_{2} !} \int_{R^{n}} \frac{(x-y)^{\alpha_{1}+\alpha_{2}} D^{\alpha_{1}} \tilde{b}_{1}(y) D^{\alpha_{2}} \tilde{b}_{2}(y)}{|x-y|^{m-2}} K(x, y) f_{1}(y) d y \\
& +\int_{R^{n}} \frac{\prod_{j=1}^{2} R_{m_{j}+1}\left(\tilde{b}_{j} ; x, y\right)}{|x-y|^{m-2}} K(x, y) f_{2}(y) d y,
\end{aligned}
$$

then

$$
\begin{aligned}
& \frac{1}{|Q|} \int_{Q}\left|T^{b}(f)(x)-T^{\tilde{b}}\left(f_{2}\right)\left(x_{0}\right)\right| d x \\
& \leq \frac{1}{|Q|} \int_{Q}\left|\int_{R^{n}} \frac{\prod_{j=1}^{2} R_{m_{j}}\left(\tilde{b}_{j} ; x, y\right)}{|x-y|^{m-2}} K(x, y) f_{1}(y) d y\right| d x \\
& \quad+\frac{C}{|Q|} \int_{Q}\left|\sum_{\left|\alpha_{1}\right|=m_{1}} \int_{R^{n}} \frac{R_{m_{2}}\left(\tilde{b}_{2} ; x, y\right)(x-y)^{\alpha_{1}}}{|x-y|^{m-2}} D^{\alpha_{1}} \tilde{b}_{1}(y) K(x, y) f_{1}(y) d y\right| d x \\
& \quad+\frac{C}{|Q|} \int_{Q}\left|\sum_{\left|\alpha_{2}\right|=m_{2}} \int_{R^{n}} \frac{R_{m_{1}}\left(\tilde{b}_{1} ; x, y\right)(x-y)^{\alpha_{2}}}{|x-y|^{m-2}} D^{\alpha_{2}} \tilde{b}_{2}(y) K(x, y) f_{1}(y) d y\right| d x \\
& \quad+\left.\frac{C}{|Q|} \int_{Q}\right|_{\left|\alpha_{1}\right|=m_{1},\left|\alpha_{2}\right|=m_{2}} \int_{R^{n}} \frac{(x-y)^{\alpha_{1}+\alpha_{2}} D^{\alpha_{1}} \tilde{b}_{1}(y) D^{\alpha_{2}} \tilde{b}_{2}(y)}{|x-y|^{m-2}} K(x, y) f_{1}(y) d y \mid d x \\
& \quad+\frac{1}{|Q|} \int_{Q}\left|T^{\tilde{b}}\left(f_{2}\right)(x)-T^{\tilde{b}}\left(f_{2}\right)\left(x_{0}\right)\right| d x:=I_{1}+I_{2}+I_{3}+I_{4}+I_{5} .
\end{aligned}
$$

Now, let us estimate $I_{1}, I_{2}, I_{3}, I_{4}$ and $I_{5}$, respectively. First, by Lemmas 1 and 5 , we get

$$
\begin{aligned}
\left|R_{m}\left(\tilde{b}_{j} ; x, y\right)\right| & \leq C|x-y|^{m} \sum_{|\alpha|=m} \sup _{x \in \tilde{Q}}\left|D^{\alpha} b_{j}(x)-\left(D^{\alpha} b_{j}\right)_{\tilde{Q}}\right| \\
& \leq C|x-y|^{m} \frac{w(\tilde{Q})^{1+\beta / n}}{|Q|} \sum_{|\alpha|=m}\left\|D^{\alpha} b_{j}\right\|_{\operatorname{Lip}_{\beta}(w)} .
\end{aligned}
$$

Thus, by the $\left(L^{s}, L^{t}\right)$-boundedness of $T$ with $1<s<n / \delta$ and $1 / t=1 / s-\delta / n$, we obtain

$$
\begin{aligned}
I_{1} & \leq C \prod_{j=1}^{2}\left(\sum_{\left|\alpha_{j}\right|=m_{j}}\left\|D^{\alpha_{j}} b_{j}\right\|_{\operatorname{Lip}_{\beta}(w)}\right)\left(\frac{|Q|^{2 / n} w(\tilde{Q})^{2+2 \beta / n}}{|Q|^{2}}\right) \frac{1}{|Q|} \int_{Q}\left|T\left(f_{1}\right)(x)\right| d x \\
& \leq C \prod_{j=1}^{2}\left(\sum_{\left|\alpha_{j}\right|=m_{j}}\left\|D^{\alpha_{j}} b_{j}\right\|_{\operatorname{Lip}_{\beta}(w)}\right)\left(\frac{|Q|^{2 / n} w(\tilde{Q})^{2+2 \beta / n}}{|Q|^{2}}\right)\left(\frac{1}{|Q|} \int_{R^{n}}\left|T\left(f_{1}\right)(x)\right|^{t} d x\right)^{1 / t}
\end{aligned}
$$




$$
\begin{aligned}
& \leq C \prod_{j=1}^{2}\left(\sum_{\left|\alpha_{j}\right|=m_{j}}\left\|D^{\alpha_{j}} b_{j}\right\|_{\operatorname{Lip}_{\beta}(w)}\right)\left(\frac{|Q|^{2 / n} w(\tilde{Q})^{2+2 \beta / n}}{|Q|^{2}}\right)|Q|^{-1 / t}\left(\int_{R^{n}}\left|f_{1}(x)\right|^{s} d x\right)^{1 / s} \\
& \leq C \prod_{j=1}^{2}\left(\sum_{\left|\alpha_{j}\right|=m_{j}}\left\|D^{\alpha_{j}} b_{j}\right\|_{\operatorname{Lip}_{\beta}(w)}\right)\left(\frac{w(\tilde{Q})}{|\tilde{Q}|}\right)^{2+2 \beta / n}\left(\frac{1}{|\tilde{Q}|^{1-s(\delta+2+2 \beta) / n}} \int_{\tilde{Q}}|f(y)|^{s} d y\right)^{1 / s} \\
& \leq C \prod_{j=1}^{2}\left(\sum_{\left|\alpha_{j}\right|=m_{j}}\left\|D^{\alpha_{j}} b_{j}\right\|_{\operatorname{Lip}_{\beta}(w)}\right) w(\tilde{x})^{2+2 \beta / n} M_{\delta+2+2 \beta, s}(f)(\tilde{x}) .
\end{aligned}
$$

For $I_{2}$, by the $\left(L^{s}, L^{t}\right)$-boundedness of $T$ with $1<s<n / \delta, 1 / t=1 / s-\delta / n$ and Lemma 5 , we get

$$
\begin{aligned}
I_{2} \leq & C \sum_{\left|\alpha_{2}\right|=m_{2}}\left\|D^{\alpha_{2}} b_{2}\right\|_{\operatorname{Lip}_{\beta}(w)} \frac{|Q|^{2 / n} w(\tilde{Q})^{1+\beta / n}}{|Q|} \sum_{\left|\alpha_{1}\right|=m_{1}} \frac{1}{|Q|} \int_{Q}\left|T\left(D^{\alpha_{1}} \tilde{b}_{1} f_{1}\right)(x)\right| d x \\
\leq & C \sum_{\left|\alpha_{2}\right|=m_{2}}\left\|D^{\alpha_{2}} b_{2}\right\|_{\operatorname{Lip}_{\beta}(w)} \frac{|Q|^{2 / n} w(\tilde{Q})^{1+\beta / n}}{|Q|} \\
& \times \sum_{\left|\alpha_{1}\right|=m_{1}}\left(\frac{1}{|Q|} \int_{R^{n}}\left|T\left(D^{\alpha_{1}} \tilde{b}_{1} f_{1}\right)(x)\right|^{t} d x\right)^{1 / t} \\
\leq & C \sum_{\left|\alpha_{2}\right|=m_{2}}\left\|D^{\alpha_{2}} b_{2}\right\|_{\operatorname{Lip}_{\beta}(w)} \frac{|Q|^{2 / n} w(\tilde{Q})^{1+\beta / n}}{|Q|}|Q|^{-1 / t} \\
& \times \sum_{\left|\alpha_{1}\right|=m_{1}}\left(\int_{R^{n}}\left|D^{\alpha_{1}} b_{1}(x)-\left(D^{\alpha} b_{1}\right)_{\tilde{Q}}\right|^{s}\left|f_{1}(x)\right|^{s} d x\right)^{1 / s} \\
\leq & C \prod_{j=1}^{2}\left(\sum_{\left|\alpha_{j}\right|=m_{j}}\left\|D^{\alpha_{j}} b_{j}\right\|_{\operatorname{Lip}_{\beta}(w)}\right) \frac{|Q|^{2 / n} w(\tilde{Q})^{2+2 \beta / n}}{|Q|^{2}}|Q|^{-1 / t}\left(\frac{1}{|\tilde{Q}|} \int_{\tilde{Q}}|f(y)|^{s} d y\right)^{1 / s} \\
\leq & C \prod_{j=1}^{2}\left(\sum_{\left|\alpha_{j}\right|=m_{j}}\left\|D^{\alpha_{j}} b_{j}\right\|_{\operatorname{Lip}_{\beta}(w)}\right)\left(\frac{w(\tilde{Q})}{|\tilde{Q}|}\right)^{2+2 \beta / n}\left(\frac{1}{|\tilde{Q}|^{1-s(\delta+2+2 \beta) / n}} \int_{\tilde{Q}}|f(y)|^{s} d y\right)^{1 / s} \\
\leq & C \prod_{j=1}^{2}\left(\sum_{\left|\alpha_{j}\right|=m_{j}}\left\|D^{\alpha_{j}} b_{j}\right\|_{\operatorname{Lip}_{\beta}(w)}\right) w(\tilde{x})^{2+2 \beta / n} M_{\delta+2+2 \beta, s}(f)(\tilde{x}) .
\end{aligned}
$$

For $I_{3}$, similar to the proof of $I_{2}$, we get

$$
I_{3} \leq C \prod_{j=1}^{2}\left(\sum_{\left|\alpha_{j}\right|=m_{j}}\left\|D^{\alpha_{j}} b_{j}\right\|_{\operatorname{Lip}_{\beta}(w)}\right) w(\tilde{x})^{2+2 \beta / n} M_{\delta+2+2 \beta, s}(f)(\tilde{x}) .
$$

Similarly, for $I_{4}$, we obtain, for $1<s<n / \delta$ and $1 / t=1 / s-\delta / n$,

$$
\begin{aligned}
I_{4} & \leq C \sum_{\left|\alpha_{1}\right|=m_{1},\left|\alpha_{2}\right|=m_{2}} \frac{|Q|^{2 / n}}{|Q|} \int_{Q}\left|T\left(D^{\alpha_{1}} \tilde{b}_{1} D^{\alpha_{2}} \tilde{b}_{2} f_{1}\right)(x)\right| d x \\
& \leq C \sum_{\left|\alpha_{1}\right|=m_{1},\left|\alpha_{2}\right|=m_{2}}|Q|^{2 / n}\left(\frac{1}{|Q|} \int_{R^{n}}\left|T\left(D^{\alpha_{1}} \tilde{b}_{1} D^{\alpha_{2}} \tilde{b}_{2} f_{1}\right)(x)\right|^{t} d x\right)^{1 / t}
\end{aligned}
$$


Chou et al. Journal of Inequalities and Applications 2013, 2013:303

Page 7 of 15

http://www.journalofinequalitiesandapplications.com/content/2013/1/303

$$
\begin{aligned}
& \leq C \sum_{\left|\alpha_{1}\right|=m_{1},\left|\alpha_{2}\right|=m_{2}}|Q|^{2 / n}|Q|^{-1 / t}\left(\int_{R^{n}}\left|D^{\alpha_{1}} \tilde{b}_{1}(x) D^{\alpha_{2}} \tilde{b}_{2}(x)\right|^{s}\left|f_{1}(x)\right|^{s} d x\right)^{1 / s} \\
& \leq C \prod_{j=1}^{2}\left(\sum_{\left|\alpha_{j}\right|=m_{j}}\left\|D^{\alpha_{j}} b_{j}\right\|_{\operatorname{Lip}_{\beta}(w)}\right) \frac{|Q|^{2 / n} w(\tilde{Q})^{2+2 \beta / n}}{|Q|^{2}}|Q|^{-1 / t}\left(\frac{1}{|\tilde{Q}|} \int_{\tilde{Q}}|f(y)|^{s} d y\right)^{1 / s} \\
& \leq C \prod_{j=1}^{2}\left(\sum_{\left|\alpha_{j}\right|=m_{j}}\left\|D^{\alpha_{j}} b_{j}\right\|_{\operatorname{Lip}_{\beta}(w)}\right)\left(\frac{w(\tilde{Q})}{|\tilde{Q}|}\right)^{2+2 \beta / n}\left(\frac{1}{|\tilde{Q}|^{1-s(\delta+2+2 \beta) / n}} \int_{\tilde{Q}}|f(y)|^{s} d y\right)^{1 / s} \\
& \leq C \prod_{j=1}^{2}\left(\sum_{\left|\alpha_{j}\right|=m_{j}}\left\|D^{\alpha_{j}} b_{j}\right\|_{\operatorname{Lip}_{\beta}(w)}\right) w(\tilde{x})^{2+2 \beta / n} M_{\delta+2+2 \beta, s}(f)(\tilde{x}) .
\end{aligned}
$$

For $I_{5}$, we write

$$
\begin{aligned}
& T^{\tilde{b}}\left(f_{2}\right)(x)-T^{\tilde{b}}\left(f_{2}\right)\left(x_{0}\right) \\
& =\int_{R^{n}}\left(\frac{K(x, y)}{|x-y|^{m}}-\frac{K\left(x_{0}, y\right)}{\left|x_{0}-y\right|^{m}}\right) \prod_{j=1}^{2} R_{m_{j}}\left(\tilde{b}_{j} ; x, y\right) f_{2}(y) d y \\
& +\int_{R^{n}}\left(R_{m_{1}}\left(\tilde{b}_{1} ; x, y\right)-R_{m_{1}}\left(\tilde{b}_{1} ; x_{0}, y\right)\right) \frac{R_{m_{2}}\left(\tilde{b}_{2} ; x, y\right)}{\left|x_{0}-y\right|^{m}} K\left(x_{0}, y\right) f_{2}(y) d y \\
& +\int_{R^{n}}\left(R_{m_{2}}\left(\tilde{b}_{2} ; x, y\right)-R_{m_{2}}\left(\tilde{b}_{2} ; x_{0}, y\right)\right) \frac{R_{m_{1}}\left(\tilde{b}_{1} ; x_{0}, y\right)}{\left|x_{0}-y\right|^{m}} K\left(x_{0}, y\right) f_{2}(y) d y \\
& -\sum_{\left|\alpha_{1}\right|=m_{1}} \frac{1}{\alpha_{1} !} \int_{R^{n}} D^{\alpha_{1}} \tilde{b}_{1}(y) f_{2}(y) \\
& \times\left[\frac{R_{m_{2}}\left(\tilde{b}_{2} ; x, y\right)(x-y)^{\alpha_{1}}}{|x-y|^{m}} K(x, y)-\frac{R_{m_{2}}\left(\tilde{b}_{2} ; x_{0}, y\right)\left(x_{0}-y\right)^{\alpha_{1}}}{\left|x_{0}-y\right|^{m}} K\left(x_{0}, y\right)\right] d y \\
& -\sum_{\left|\alpha_{2}\right|=m_{2}} \frac{1}{\alpha_{2} !} \int_{R^{n}} D^{\alpha_{2}} \tilde{b}_{2}(y) f_{2}(y) \\
& \times\left[\frac{R_{m_{1}}\left(\tilde{b}_{1} ; x, y\right)(x-y)^{\alpha_{2}}}{|x-y|^{m}} K(x, y)-\frac{R_{m_{1}}\left(\tilde{b}_{1} ; x_{0}, y\right)\left(x_{0}-y\right)^{\alpha_{2}}}{\left|x_{0}-y\right|^{m}} K\left(x_{0}, y\right)\right] d y \\
& +\sum_{\left|\alpha_{1}\right|=m_{1},\left|\alpha_{2}\right|=m_{2}} \frac{1}{\alpha_{1} ! \alpha_{2} !} \int_{R^{n}}\left[\frac{(x-y)^{\alpha_{1}+\alpha_{2}}}{|x-y|^{m}} K(x, y)-\frac{\left(x_{0}-y\right)^{\alpha_{1}+\alpha_{2}}}{\left|x_{0}-y\right|^{m}} K\left(x_{0}, y\right)\right] \\
& \times D^{\alpha_{1}} \tilde{b}_{1}(y) D^{\alpha_{2}} \tilde{b}_{2}(y) f_{2}(y) d y \\
& =I_{5}^{(1)}+I_{5}^{(2)}+I_{5}^{(3)}+I_{5}^{(4)}+I_{5}^{(5)}+I_{5}^{(6)} \text {. }
\end{aligned}
$$

Note that $|x-y| \sim\left|x_{0}-y\right|$ for $x \in Q$ and $y \in R^{n} \backslash \tilde{Q}=\bigcup_{l=0}^{\infty}\left(2^{l+1} \tilde{Q} \backslash 2^{l} \tilde{Q}\right)$. By Lemmas 1 and 5, we get

$$
\left|R_{m_{j}}\left(\tilde{b}_{j} ; x, y\right)\right| \leq C \sum_{|\alpha|=m_{j}}\left\|D^{\alpha} b_{j}\right\|_{\operatorname{Lip}_{\beta}(w)}\left(\frac{w\left(2^{l+1} \tilde{Q}\right)^{1+\beta / n}}{\left|2^{l+1} Q\right|}\right)|x-y|^{m_{j}} .
$$


Then, by the conditions on $K$, we obtain

$$
\begin{aligned}
& \left|I_{5}^{(1)}\right| \leq C \int_{R^{n} \backslash \tilde{Q}}\left(\frac{\left|x-x_{0}\right|}{\left|x_{0}-y\right|^{m+n-1-\delta}}+\frac{\left|x-x_{0}\right|^{\varepsilon}}{\left|x_{0}-y\right|^{m+n+\varepsilon-2-\delta}}\right) \prod_{j=1}^{2}\left|R_{m_{j}}\left(\tilde{b}_{j} ; x, y\right)\right||f(y)| d y \\
& \leq C \prod_{j=1}^{2}\left(\sum_{\left|\alpha_{j}\right|=m_{j}}\left\|D^{\alpha_{j}} b_{j}\right\|_{\operatorname{Lip}_{\beta}(w)}\right) \sum_{l=0}^{\infty} \frac{w\left(2^{l+1} \tilde{Q}\right)^{2+2 \beta / n}}{\left|2^{l+1} Q\right|^{2}} \\
& \times \int_{2^{l+1} \tilde{Q} \backslash 2^{l} \tilde{Q}}\left(\frac{\left|x-x_{0}\right|}{\left|x_{0}-y\right|^{n-1-\delta}}+\frac{\left|x-x_{0}\right|^{\varepsilon}}{\left|x_{0}-y\right|^{n+\varepsilon-2-\delta}}\right)|f(y)| d y \\
& \leq C \prod_{j=1}^{2}\left(\sum_{\left|\alpha_{j}\right|=m_{j}}\left\|D^{\alpha_{j}} b_{j}\right\|_{\operatorname{Lip}_{\beta}(w)}\right) \sum_{l=0}^{\infty} \frac{w\left(2^{l+1} \tilde{Q}\right)^{2+2 \beta / n}}{\left|2^{l+1} Q\right|^{2}} \\
& \times \int_{2^{l+1} \tilde{Q} \backslash 2^{l} \tilde{Q}}\left(\frac{d}{\left(2^{l} d\right)^{n-1-\delta}}+\frac{d^{\varepsilon}}{\left(2^{l} d\right)^{n+\varepsilon-2-\delta}}\right)|f(y)| d y \\
& \leq C \prod_{j=1}^{2}\left(\sum_{\left|\alpha_{j}\right|=m_{j}}\left\|D^{\alpha_{j}} b_{j}\right\|_{\operatorname{Lip}_{\beta}(w)}\right) \sum_{l=0}^{\infty}\left(\frac{w\left(2^{l+1} \tilde{Q}\right)}{\left|2^{l+1} \tilde{Q}\right|}\right)^{2+2 \beta / n}\left(2^{-l}+2^{-l \varepsilon}\right) \\
& \times \frac{1}{\left|2^{l+1} \tilde{Q}\right|^{1-(\delta+2+2 \beta) / n}} \int_{2^{l+1} \tilde{Q}}|f(y)| d y \\
& \leq C \prod_{j=1}^{2}\left(\sum_{\left|\alpha_{j}\right|=m_{j}}\left\|D^{\alpha_{j}} b_{j}\right\|_{\operatorname{Lip}_{\beta}(w)}\right) w(\tilde{x})^{2+2 \beta / n} \sum_{l=0}^{\infty}\left(2^{-l}+2^{-l \varepsilon}\right) \\
& \times\left(\frac{1}{\left|2^{l+1} \tilde{Q}\right|^{1-s(\delta+2+2 \beta) / n}} \int_{2^{l+1} \tilde{Q}}|f(y)|^{s} d y\right)^{1 / s} \\
& \leq C \prod_{j=1}^{2}\left(\sum_{\left|\alpha_{j}\right|=m_{j}}\left\|D^{\alpha_{j}} b_{j}\right\|_{\operatorname{Lip}_{\beta}(w)}\right) w(\tilde{x})^{2+2 \beta / n} M_{\delta+2+2 \beta, s}(f)(\tilde{x}) \sum_{l=1}^{\infty}\left(2^{-l}+2^{-l \varepsilon}\right) \\
& \leq C \prod_{j=1}^{2}\left(\sum_{\left|\alpha_{j}\right|=m_{j}}\left\|D^{\alpha_{j}} b_{j}\right\|_{\operatorname{Lip}_{\beta}(w)}\right) w(\tilde{x})^{2+2 \beta / n} M_{\delta+2+2 \beta, s}(f)(\tilde{x}) .
\end{aligned}
$$

For $I_{5}^{(2)}$, by the formula (see [1])

$$
R_{m_{j}}\left(\tilde{b}_{j} ; x, y\right)-R_{m_{j}}\left(\tilde{b}_{j} ; x_{0}, y\right)=\sum_{|\eta|<m_{j}} \frac{1}{\eta !} R_{m_{j}-|\eta|}\left(D^{\eta} \tilde{b}_{j} ; x, x_{0}\right)(x-y)^{\eta}
$$

and Lemma 5, we get

$$
\begin{aligned}
\left|I_{5}^{(2)}\right| \leq & C \prod_{j=1}^{2}\left(\sum_{|\alpha|=m_{j}}\left\|D^{\alpha} b_{j}\right\|_{\operatorname{Lip}_{\beta}(w)}\right) \\
& \times \sum_{l=0}^{\infty} \frac{w\left(2^{l+1} Q\right)^{2+2 \beta / n}}{\left|2^{l+1} Q\right|^{2}} \int_{2^{l+1} \tilde{Q} \backslash 2^{l} \tilde{Q}} \frac{\left|x-x_{0}\right|}{\left|x_{0}-y\right|^{n-1-\delta}}|f(y)| d y \\
\leq & C \prod_{j=1}^{2}\left(\sum_{|\alpha|=m_{j}}\left\|D^{\alpha} b_{j}\right\|_{\operatorname{Lip}_{\beta}(w)}\right) w(\tilde{x})^{2+2 \beta / n} M_{\delta+2+2 \beta, s}(f)(\tilde{x}) .
\end{aligned}
$$


Similarly,

$$
\left|I_{5}^{(3)}\right| \leq C \prod_{j=1}^{2}\left(\sum_{|\alpha|=m_{j}}\left\|D^{\alpha} b_{j}\right\|_{\operatorname{Lip}_{\beta}(w)}\right) w(\tilde{x})^{2+2 \beta / n} M_{\delta+2+2 \beta, s}(f)(\tilde{x}) .
$$

For $I_{5}^{(4)}$, similar to the estimates of $I_{5}^{(1)}$ and $I_{5}^{(2)}$, and noticing that for $b \in \operatorname{Lip}_{\beta}(w), w \in A_{1}$ and $x \in Q$, by Lemma 4 , we have

$$
\left|b_{Q}-b_{2^{l} Q}\right| \leq C l w(x) w\left(2^{l} Q\right)^{\beta / n}\|b\|_{\operatorname{Lip}_{\beta}(w)} .
$$

Thus, we obtain

$$
\begin{aligned}
& \left|I_{5}^{(4)}\right| \leq C \sum_{\left|\alpha_{1}\right|=m_{1}} \int_{R^{n} \backslash \tilde{Q}}\left|\frac{(x-y)^{\alpha_{1}} K(x, y)}{|x-y|^{m-2}}-\frac{\left(x_{0}-y\right)^{\alpha_{1}} K\left(x_{0}, y\right)}{\left|x_{0}-y\right|^{m-2}}\right| \\
& \times\left|R_{m_{2}}\left(\tilde{b}_{2} ; x, y\right)\right|\left|D^{\alpha_{1}} \tilde{b}_{1}(y)\right||f(y)| d y \\
& +C \sum_{\left|\alpha_{1}\right|=m_{1}} \int_{R^{n} \backslash \tilde{Q}}\left|R_{m_{2}}\left(\tilde{b}_{2} ; x, y\right)-R_{m_{2}}\left(\tilde{b}_{2} ; x_{0}, y\right)\right| \\
& \times \frac{\left|\left(x_{0}-y\right)^{\alpha_{1}} K\left(x_{0}, y\right)\right|}{\left|x_{0}-y\right|^{m-2}}\left|D^{\alpha_{1}} \tilde{b}_{1}(y) f(y)\right| d y \\
& \leq C \sum_{\left|\alpha_{2}\right|=m_{2}}\left\|D^{\alpha_{2}} b_{2}\right\|_{\operatorname{Lip}_{\beta}(w)} \sum_{|\alpha|=m_{1}} \sum_{l=0}^{\infty} \int_{2^{l+1} \tilde{Q} \backslash 2^{l} \tilde{Q}}\left(\frac{\left|x-x_{0}\right|}{\left|x_{0}-y\right|^{n-1-\delta}}+\frac{\left|x-x_{0}\right|^{\varepsilon}}{\left|x_{0}-y\right|^{n+\varepsilon-2-\delta}}\right) \\
& \times \frac{w\left(2^{l+1} \tilde{Q}\right)^{1+\beta / n}}{\left|2^{l+1} \tilde{Q}\right|}\left(\left|D^{\alpha_{1}} b_{1}(y)-\left(D^{\alpha_{1}} b_{1}\right)_{2^{l+1} \tilde{Q}}\right|\right. \\
& \left.+\left|\left(D^{\alpha_{1}} b_{1}\right)_{2^{l+1} \tilde{Q}}-\left(D^{\alpha_{1}} b_{1}\right)_{\tilde{Q}}\right|\right)|f(y)| d y \\
& \leq C \prod_{j=1}^{2}\left(\sum_{|\alpha|=m_{j}}\left\|D^{\alpha} b_{j}\right\|_{\operatorname{Lip}_{\beta}(w)}\right) \sum_{l=0}^{\infty} \int_{2^{l+1} \tilde{Q} \backslash 2^{2} \tilde{Q}}\left(\frac{d}{\left(2^{l} d\right)^{n-1-\delta}}+\frac{d^{\varepsilon}}{\left(2^{l} d\right)^{n+\varepsilon-2-\delta}}\right) \\
& \times \frac{w\left(2^{l+1} \tilde{Q}\right)^{1+\beta / n}}{\left|2^{l+1} \tilde{Q}\right|}\left[\frac{w\left(2^{l+1} \tilde{Q}\right)^{1+\beta / n}}{\left|2^{l+1} \tilde{Q}\right|}+l w(\tilde{x}) w\left(2^{l+1} \tilde{Q}\right)^{\beta / n}\right]|f(y)| d y \\
& \leq C \prod_{j=1}^{2}\left(\sum_{|\alpha|=m_{j}}\left\|D^{\alpha} b_{j}\right\|_{\operatorname{Lip}_{\beta}(w)}\right) \sum_{l=0}^{\infty} w(\tilde{x})\left(\frac{w\left(2^{l+1} \tilde{Q}\right)}{\left|2^{l+1} \tilde{Q}\right|}\right)^{1+2 \beta / n} \\
& \times\left(\frac{1}{\left|2^{l+1} \tilde{Q}\right|^{1-s(\delta+2+2 \beta) / n}} \int_{2^{l+1} \tilde{Q}}|f(y)|^{s} d y\right)^{1 / s} l\left(2^{-l}+2^{-l \varepsilon}\right) \\
& \leq C \prod_{j=1}^{2}\left(\sum_{|\alpha|=m_{j}}\left\|D^{\alpha} b_{j}\right\|_{\operatorname{Lip}_{\beta}(w)}\right) w(\tilde{x})^{2+2 \beta / n} M_{\delta+2+2 \beta, s}(f)(\tilde{x}) .
\end{aligned}
$$

Similarly,

$$
\left|I_{5}^{(5)}\right| \leq C \prod_{j=1}^{2}\left(\sum_{|\alpha|=m_{j}}\left\|D^{\alpha} b_{j}\right\|_{\operatorname{Lip}_{\beta}(w)}\right) w(\tilde{x})^{2+2 \beta / n} M_{\delta+2+2 \beta, s}(f)(\tilde{x}) .
$$


For $I_{5}^{(6)}$, we get

$$
\begin{aligned}
\left|I_{5}^{(6)}\right| \leq & C \sum_{\left|\alpha_{1}\right|=m_{1},\left|\alpha_{2}\right|=m_{2}} \int_{R^{n} \backslash \tilde{Q}}\left|\frac{(x-y)^{\alpha_{1}+\alpha_{2}} K(x, y)}{|x-y|^{m}}-\frac{\left(x_{0}-y\right)^{\alpha_{1}+\alpha_{2}} K\left(x_{0}, y\right)}{\left|x_{0}-y\right|^{m}}\right| \\
& \times\left(\left|D^{\alpha_{1}} b_{1}(y)-\left(D^{\alpha_{1}} b_{1}\right)_{2^{l+1} \tilde{Q}}\right|+\left|\left(D^{\alpha_{1}} b_{1}\right)_{2^{l+1} \tilde{Q}}-\left(D^{\alpha_{1}} b_{1}\right)_{\tilde{Q}}\right|\right) \\
& \times\left(\left|D^{\alpha_{2}} b_{2}(y)-\left(D^{\alpha_{2}} b_{2}\right)_{2^{l+1} \tilde{Q}}\right|+\left|\left(D^{\alpha_{2}} b_{2}\right)_{2^{l+1} \tilde{Q}}-\left(D^{\alpha_{2}} b_{2}\right)_{\tilde{Q}}\right|\right)|f(y)| d y \\
\leq & C \prod_{j=1}^{2}\left(\sum_{\left|\alpha_{j}\right|=m_{j}}\left\|D^{\alpha_{j}} b_{j}\right\|_{\operatorname{Lip}_{\beta}(w)}\right) \sum_{l=0}^{\infty} \int_{2^{l+1} \tilde{Q} \mid 2^{l} \tilde{Q}}\left(\frac{\left|x-x_{0}\right|}{\left|x_{0}-y\right|^{n-1-\delta}}+\frac{\left|x-x_{0}\right|^{\varepsilon}}{\left|x_{0}-y\right|^{n+\varepsilon-2-\delta}}\right) \\
& \times\left[\frac{w\left(2^{l+1} \tilde{Q}\right)^{1+\beta / n}}{\left|2^{l+1} \tilde{Q}\right|}+l w(\tilde{x}) w\left(2^{l+1} \tilde{Q}\right)^{\beta / n}\right]^{2}|f(y)| d y \\
\leq & C \prod_{j=1}^{2}\left(\sum_{\left|\alpha_{j}\right|=m_{j}}\left\|D^{\alpha_{j}} b_{j}\right\|_{\operatorname{Lip}_{\beta}(w)}\right) \sum_{l=0}^{\infty}\left[w(\tilde{x})\left(\frac{w\left(2^{l+1} \tilde{Q}\right)}{\left|2^{l+1} \tilde{Q}\right|}\right)^{\beta / n}\right]^{2} \\
& \times\left(\left.\frac{1}{\left|2^{l+1} \tilde{Q}\right|^{1-s(\delta+2+2 \beta) / n}} \int_{2^{l+1} \tilde{Q}} f f(y)\right|^{s} d y\right)^{1 / s} l^{2}\left(2^{-l}+2^{-l \varepsilon}\right) \\
\leq & C \prod_{j=1}^{2}\left(\sum_{\left|\alpha_{j}\right|=m_{j}}\left\|D^{\alpha_{j}} b_{j}\right\|_{\operatorname{Lip}_{\beta}(w)}\right) w(\tilde{x})^{2+2 \beta / n} M_{\delta+2+2 \beta, s}(f)(\tilde{x}) .
\end{aligned}
$$

Thus

$$
\left|T^{\tilde{b}}\left(f_{2}\right)(x)-T^{\tilde{b}}\left(f_{2}\right)\left(x_{0}\right)\right| \leq C \prod_{j=1}^{2}\left(\sum_{|\alpha|=m_{j}}\left\|D^{\alpha} b_{j}\right\|_{\operatorname{Lip}_{\beta}(w)}\right) w(\tilde{x})^{2+2 \beta / n} M_{\delta+2+2 \beta, s}(f)(\tilde{x})
$$

and

$$
I_{5} \leq C \prod_{j=1}^{2}\left(\sum_{\left|\alpha_{j}\right|=m_{j}}\left\|D^{\alpha_{j}} b_{j}\right\|_{\operatorname{Lip}_{\beta}(w)}\right) w(\tilde{x})^{2+2 \beta / n} M_{\delta+2+2 \beta, s}(f)(\tilde{x}) .
$$

This completes the proof of the lemma.

Proof of Key Lemma 2 Without loss of generality, we may assume $k=2$. By using the same argument as in the proof of Key Lemma 1, we have

$$
\begin{aligned}
& \frac{1}{|Q|^{1+2 \beta / n}} \int_{Q}\left|T^{b}(f)(x)-T^{\tilde{b}}\left(f_{2}\right)\left(x_{0}\right)\right| d x \\
& \leq \frac{1}{|Q|^{1+2 \beta / n}} \int_{Q}\left|\int_{R^{n}} \frac{\prod_{j=1}^{2} R_{m_{j}}\left(\tilde{b}_{j} ; x, y\right)}{|x-y|^{m-2}} K(x, y) f_{1}(y) d y\right| d x \\
& \quad+\frac{C}{|Q|^{1+2 \beta / n}} \int_{Q}\left|\sum_{\left|\alpha_{1}\right|=m_{1}} \int_{R^{n}} \frac{R_{m_{2}}\left(\tilde{b}_{2} ; x, y\right)(x-y)^{\alpha_{1}}}{|x-y|^{m-2}} D^{\alpha_{1}} \tilde{b}_{1}(y) K(x, y) f_{1}(y) d y\right| d x \\
& \quad+\frac{C}{|Q|^{1+2 \beta / n}} \int_{Q}\left|\sum_{\left|\alpha_{2}\right|=m_{2}} \int_{R^{n}} \frac{R_{m_{1}}\left(\tilde{b}_{1} ; x, y\right)(x-y)^{\alpha_{2}}}{|x-y|^{m-2}} D^{\alpha_{2}} \tilde{b}_{2}(y) K(x, y) f_{1}(y) d y\right| d x
\end{aligned}
$$


Chou et al. Journal of Inequalities and Applications 2013, 2013:303

Page 11 of 15

http://www.journalofinequalitiesandapplications.com/content/2013/1/303

$$
\begin{aligned}
& +\left.\frac{C}{|Q|^{1+2 \beta / n}} \int_{Q}\right|_{\left|\alpha_{1}\right|=m_{1},\left|\alpha_{2}\right|=m_{2}} \int_{R^{n}} \frac{(x-y)^{\alpha_{1}+\alpha_{2}} D^{\alpha_{1}} \tilde{b}_{1}(y) D^{\alpha_{2}} \tilde{b}_{2}(y)}{|x-y|^{m-2}} K(x, y) f_{1}(y) d y \mid d x \\
& +\frac{1}{|Q|^{1+2 \beta / n}} \int_{Q}\left|T^{\tilde{b}}\left(f_{2}\right)(x)-T^{\tilde{b}}\left(f_{2}\right)\left(x_{0}\right)\right| d x \\
& :=J_{1}+J_{2}+J_{3}+J_{4}+J_{5} .
\end{aligned}
$$

We obtain

$$
\begin{aligned}
& J_{1} \leq C \prod_{j=1}^{2}\left(\sum_{\left|\alpha_{j}\right|=m_{j}}\left\|D^{\alpha_{j}} b_{j}\right\|_{\operatorname{Lip}_{\beta}(w)}\right)|Q|^{2 / n}\left(\frac{w(\tilde{Q})}{|Q|}\right)^{2+2 \beta / n}\left(\frac{1}{|Q|} \int_{R^{n}}\left|T\left(f_{1}\right)(x)\right|^{t} d x\right)^{1 / t} \\
& \leq C \prod_{j=1}^{2}\left(\sum_{\left|\alpha_{j}\right|=m_{j}}\left\|D^{\alpha_{j}} b_{j}\right\|_{\operatorname{Lip}_{\beta}(w)}\right)|Q|^{2 / n}\left(\frac{w(\tilde{Q})}{|Q|}\right)^{2+2 \beta / n}|Q|^{-1 / t}\left(\int_{R^{n}}\left|f_{1}(x)\right|^{s} d x\right)^{1 / s} \\
& \leq C \prod_{j=1}^{2}\left(\sum_{\left|\alpha_{j}\right|=m_{j}}\left\|D^{\alpha_{j}} b_{j}\right\|_{\operatorname{Lip}_{\beta}(w)}\right)\left(\frac{w(\tilde{Q})}{|\tilde{Q}|}\right)^{2+2 \beta / n}\left(\frac{1}{|\tilde{Q}|^{1-s(\delta+2) / n}} \int_{\tilde{Q}}|f(y)|^{s} d y\right)^{1 / s} \\
& \leq C \prod_{j=1}^{2}\left(\sum_{\left|\alpha_{j}\right|=m_{j}}\left\|D^{\alpha_{j}} b_{j}\right\|_{\operatorname{Lip}_{\beta}(w)}\right) w(\tilde{x})^{2+2 \beta / n} M_{\delta+2, s}(f)(\tilde{x}), \\
& J_{2} \leq C \sum_{\left|\alpha_{2}\right|=m_{2}}\left\|D^{\alpha_{2}} b_{2}\right\|_{\operatorname{Lip}_{\beta}(w)} \frac{|Q|^{2 / n} w(\tilde{Q})^{1+\beta / n}}{|Q|^{1+2 \beta / n}} \sum_{\left|\alpha_{1}\right|=m_{1}}\left(\frac{1}{|Q|} \int_{R^{n}}\left|T\left(D^{\alpha_{1}} \tilde{b}_{1} f_{1}\right)(x)\right|^{t} d x\right)^{1 / t} \\
& \leq C \sum_{\left|\alpha_{2}\right|=m_{2}}\left\|D^{\alpha_{2}} b_{2}\right\|_{\operatorname{Lip}_{\beta}(w)} \frac{|Q|^{2 / n} w(\tilde{Q})^{1+\beta / n}}{|Q|^{1+2 \beta / n}}|Q|^{-1 / t} \sum_{\left|\alpha_{1}\right|=m_{1}}\left(\int_{R^{n}}\left|D^{\alpha_{1}} \tilde{b}_{1}(x)\right|^{s}\left|f_{1}(x)\right|^{s} d x\right)^{1 / s} \\
& \leq C \prod_{j=1}^{2}\left(\sum_{\left|\alpha_{j}\right|=m_{j}}\left\|D^{\alpha_{j}} b_{j}\right\|_{\operatorname{Lip}_{\beta}(w)}\right)\left(\frac{w(\tilde{Q})}{|\tilde{Q}|}\right)^{2+2 \beta / n}\left(\frac{1}{|\tilde{Q}|^{1-s(\delta+2) / n}} \int_{\tilde{Q}}|f(y)|^{s} d y\right)^{1 / s} \\
& \leq C \prod_{j=1}^{2}\left(\sum_{\left|\alpha_{j}\right|=m_{j}}\left\|D^{\alpha_{j}} b_{j}\right\|_{\operatorname{Lip}_{\beta}(w)}\right) w(\tilde{x})^{2+2 \beta / n} M_{\delta+2, s}(f)(\tilde{x}), \\
& J_{3} \leq C \prod_{j=1}^{2}\left(\sum_{\left|\alpha_{j}\right|=m_{j}}\left\|D^{\alpha_{j}} b_{j}\right\|_{\operatorname{Lip}_{\beta}(w)}\right) w(\tilde{x})^{2+2 \beta / n} M_{\delta+2, s}(f)(\tilde{x}), \\
& J_{4} \leq C \sum_{\left|\alpha_{1}\right|=m_{1},\left|\alpha_{2}\right|=m_{2}} \frac{|Q|^{2 / n}}{|Q|^{2 \beta / n}}\left(\frac{1}{|Q|} \int_{R^{n}}\left|T\left(D^{\alpha_{1}} \tilde{b}_{1} D^{\alpha_{2}} \tilde{b}_{2} f_{1}\right)(x)\right|^{t} d x\right)^{1 / t} \\
& \leq C \sum_{\left|\alpha_{1}\right|=m_{1},\left|\alpha_{2}\right|=m_{2}} \frac{|Q|^{2 / n}}{|Q|^{2 \beta / n}}|Q|^{-1 / t}\left(\int_{R^{n}}\left|D^{\alpha_{1}} \tilde{b}_{1}(x) D^{\alpha_{2}} \tilde{b}_{2}(x)\right|^{s}\left|f_{1}(x)\right|^{s} d x\right)^{1 / s} \\
& \leq C \prod_{j=1}^{2}\left(\sum_{\left|\alpha_{j}\right|=m_{j}}\left\|D^{\alpha_{j}} b_{j}\right\|_{\operatorname{Lip}_{\beta}(w)}\right)\left(\frac{w(\tilde{Q})}{|\tilde{Q}|}\right)^{2+2 \beta / n}\left(\frac{1}{|\tilde{Q}|^{1-s(\delta+2) / n}} \int_{\tilde{Q}}|f(y)|^{s} d y\right)^{1 / s} \\
& \leq C \prod_{j=1}^{2}\left(\sum_{\left|\alpha_{j}\right|=m_{j}}\left\|D^{\alpha_{j}} b_{j}\right\|_{\operatorname{Lip}_{\beta}(w)}\right) w(\tilde{x})^{2+2 \beta / n} M_{\delta+2, s}(f)(\tilde{x}) .
\end{aligned}
$$


For $J_{5}$, similar to the proof of $I_{5}$ in Key Lemma 1, we obtain

$$
\begin{aligned}
& \left|T^{\tilde{b}}\left(f_{2}\right)(x)-T^{\tilde{b}}\left(f_{2}\right)\left(x_{0}\right)\right| \\
& \leq C \prod_{j=1}^{2}\left(\sum_{\left|\alpha_{j}\right|=m_{j}}\left\|D^{\alpha_{j}} b_{j}\right\|_{\operatorname{Lip}_{\beta}(w)}\right) \sum_{l=0}^{\infty} \frac{w\left(2^{l+1} \tilde{Q}\right)^{2+2 \beta / n}}{\left|2^{l+1} Q\right|^{2}} \\
& \times \int_{2^{l+1} \tilde{Q} \backslash 2^{l} \tilde{Q}}\left(\frac{d}{\left(2^{l} d\right)^{n-1-\delta}}+\frac{d^{\varepsilon}}{\left(2^{l} d\right)^{n+\varepsilon-2-\delta}}\right)|f(y)| d y \\
& +C \prod_{j=1}^{2}\left(\sum_{|\alpha|=m_{j}}\left\|D^{\alpha} b_{j}\right\|_{\operatorname{Lip}_{\beta}(w)}\right) \sum_{l=0}^{\infty} \frac{w\left(2^{l+1} Q\right)^{2+2 \beta / n}}{\left|2^{l+1} Q\right|^{2}} \int_{2^{l+1} \tilde{Q} \backslash 2^{l} \tilde{Q}} \frac{d}{\left(2^{l} d\right)^{n-1-\delta}}|f(y)| d y \\
& +C \prod_{j=1}^{2}\left(\sum_{|\alpha|=m_{j}}\left\|D^{\alpha} b_{j}\right\|_{\operatorname{Lip}_{\beta}(w)}\right) \sum_{l=0}^{\infty} \int_{2^{l+1} \tilde{Q} \backslash 2^{l} \tilde{Q}}\left(\frac{d}{\left(2^{l} d\right)^{n-1-\delta}}+\frac{d^{\varepsilon}}{\left(2^{l} d\right)^{n+\varepsilon-2-\delta}}\right) \\
& \times \frac{w\left(2^{l+1} \tilde{Q}\right)^{1+\beta / n}}{\left|2^{l+1} \tilde{Q}\right|}\left[\frac{w\left(2^{l+1} \tilde{Q}\right)^{1+\beta / n}}{\left|2^{l+1} \tilde{Q}\right|}+l w(\tilde{x}) w\left(2^{l+1} \tilde{Q}\right)^{\beta / n}\right]|f(y)| d y \\
& +C \prod_{j=1}^{2}\left(\sum_{\left|\alpha_{j}\right|=m_{j}}\left\|D^{\alpha_{j}} b_{j}\right\|_{\operatorname{Lip}_{\beta}(w)}\right) \sum_{l=0}^{\infty} \int_{2^{l+1} \tilde{Q} \backslash 2^{l} \tilde{Q}}\left(\frac{d}{\left(2^{l} d\right)^{n-1-\delta}}+\frac{d^{\varepsilon}}{\left(2^{l} d\right)^{n+\varepsilon-2-\delta}}\right) \\
& \times\left[\frac{w\left(2^{l+1} \tilde{Q}\right)^{1+\beta / n}}{\left|2^{l+1} \tilde{Q}\right|}+l w(\tilde{x}) w\left(2^{l+1} \tilde{Q}\right)^{\beta / n}\right]^{2}|f(y)| d y \\
& \leq C \prod_{j=1}^{2}\left(\sum_{\left|\alpha_{j}\right|=m_{j}}\left\|D^{\alpha_{j}} b_{j}\right\|_{\operatorname{Lip}_{\beta}(w)}\right)|Q|^{2 \beta / n} \sum_{l=0}^{\infty}\left(\frac{w\left(2^{l+1} \tilde{Q}\right)}{\left|2^{l+1} Q\right|}\right)^{2+2 \beta / n}\left(2^{l(2 \beta-1)}+2^{l(2 \beta-\varepsilon)}\right) \\
& \times\left(\frac{1}{\left|2^{l+1} \tilde{Q}\right|^{1-s(\delta+2) / n}} \int_{2^{l+1} \tilde{Q}}|f(y)|^{s} d y\right)^{1 / s} \\
& +C \prod_{j=1}^{2}\left(\sum_{|\alpha|=m_{j}}\left\|D^{\alpha} b_{j}\right\|_{\operatorname{Lip}_{\beta}(w)}\right)|Q|^{2 \beta / n} \sum_{l=0}^{\infty} w(\tilde{x})\left(\frac{w\left(2^{l+1} \tilde{Q}\right)}{\left|2^{l+1} \tilde{Q}\right|}\right)^{1+2 \beta / n} \\
& \times\left(\frac{1}{\left|2^{l+1} \tilde{Q}\right|^{1-s(\delta+2) / n}} \int_{2^{l+1} \tilde{Q}}|f(y)|^{s} d y\right)^{1 / s} l\left(2^{l(2 \beta-1)}+2^{l(2 \beta-\varepsilon)}\right) \\
& +C \prod_{j=1}^{2}\left(\sum_{\left|\alpha_{j}\right|=m_{j}}\left\|D^{\alpha_{j}} b_{j}\right\|_{\operatorname{Lip}_{\beta}(w)}\right)|Q|^{2 \beta / n} \sum_{l=0}^{\infty}\left[w(\tilde{x})\left(\frac{w\left(2^{l+1} \tilde{Q}\right)}{\left|2^{l+1} \tilde{Q}\right|}\right)^{\beta / n}\right]^{2} \\
& \times\left(\frac{1}{\left|2^{l+1} \tilde{Q}\right|^{1-s(\delta+2) / n}} \int_{2^{l+1} \tilde{Q}}|f(y)|^{s} d y\right)^{1 / s} l^{2}\left(2^{l(2 \beta-1)}+2^{l(2 \beta-\varepsilon)}\right) \\
& \leq C \prod_{j=1}^{2}\left(\sum_{\left|\alpha_{j}\right|=m_{j}}\left\|D^{\alpha_{j}} b_{j}\right\|_{\operatorname{Lip}_{\beta}(w)}\right)|Q|^{2 \beta / n} w(\tilde{x})^{2+2 \beta / n} M_{\delta+2, s}(f)(\tilde{x}) .
\end{aligned}
$$

Thus

$$
J_{5} \leq C \prod_{j=1}^{2}\left(\sum_{\left|\alpha_{j}\right|=m_{j}}\left\|D^{\alpha_{j}} b_{j}\right\|_{\operatorname{Lip}_{\beta}(w)}\right) w(\tilde{x})^{2+2 \beta / n} M_{\delta+2, s}(f)(\tilde{x}) .
$$

This completes the proof of the lemma. 
Proof of Theorem 1 By Key Lemma 1, we get the sharp function estimate of $T^{b}$ as follows:

$$
\left(T^{b}(f)\right)^{\#}(\tilde{x}) \leq C \prod_{j=1}^{k}\left(\sum_{\left|\alpha_{j}\right|=m_{j}}\left\|D^{\alpha_{j}} b_{j}\right\|_{\operatorname{Lip}_{\beta}(w)}\right) w(\tilde{x})^{k+k \beta / n} M_{\delta+k+k \beta, s}(f)(\tilde{x}) .
$$

Now, choose $1<s<p$ in Key Lemma 1, by using Lemma 3 and notice that $w^{1-q(k-(\delta+k) / n)} \in$ $A_{\infty}$ and $w^{1 / p} \in A(p, q)$. We get

$$
\begin{aligned}
& \left\|T^{b}(f)\right\|_{\left.L^{q\left(w^{1}-q(k-(\delta+k) / n)\right.}\right)} \\
& \quad \leq\left\|M\left(T^{b}(f)\right)\right\|_{L^{q}\left(w^{1-q(k-(\delta+k) / n)}\right)} \\
& \quad \leq C\left\|\left(T^{b}(f)\right)^{\#}\right\|_{L^{q}\left(w^{1-q(k-(\delta+k) / n)}\right)} \\
& \quad \leq C \prod_{j=1}^{k}\left(\sum_{\left|\alpha_{j}\right|=m_{j}}\left\|D^{\alpha_{j}} b_{j}\right\|_{\operatorname{Lip}_{\beta}(w)}\right)\left\|M_{\delta+k+k \beta, s}(f) w^{k+k \beta / n}\right\|_{L^{q}\left(w^{1-q(k-(\delta+k) / n)}\right)} \\
& \quad C \prod_{j=1}^{k}\left(\sum_{\left|\alpha_{j}\right|=m_{j}}\left\|D^{\alpha_{j}} b_{j}\right\|_{\operatorname{Lip}_{\beta}(w)}\right)\left\|M_{\delta+k+k \beta, s}(f)\right\|_{L^{q}\left(w^{q / p}\right)} \\
& \quad \leq C \prod_{j=1}^{k}\left(\sum_{\left|\alpha_{j}\right|=m_{j}}\left\|D^{\alpha_{j}} b_{j}\right\|_{\operatorname{Lip}_{\beta}(w)}\right)\|f\|_{L^{p}(w) .} .
\end{aligned}
$$

This completes the proof of the theorem.

Proof of Theorem 2 Choose $1<s<p$ in Key Lemma 2, notice that $w^{1-q(k-(\delta+k-k \beta) / n)} \in A_{\infty}$ and $w^{1 / p} \in A(p, q)$. By using Lemmas 2 and 3 , we obtain

$$
\begin{aligned}
& \left\|T^{b}(f)\right\|_{\dot{F}_{q}^{k \beta, \infty}}{ }_{\left(w^{1}-q(k-(\delta+k-k \beta) / n)\right.} \\
& \leq C \prod_{j=1}^{k}\left(\sum_{\left|\alpha_{j}\right|=m_{j}}\left\|D^{\alpha_{j}} b_{j}\right\|_{\operatorname{Lip}_{\beta}(w)}\right)\left\|M_{\delta+k, s}(f) w^{k+k \beta / n}\right\|_{L^{q}\left(w^{1-q(k-(\delta+k-k \beta) / n)}\right)} \\
& \quad=C \prod_{j=1}^{k}\left(\sum_{\left|\alpha_{j}\right|=m_{j}}\left\|D^{\alpha_{j}} b_{j}\right\|_{\operatorname{Lip}_{\beta}(w)}\right)\left\|M_{\delta+k, s}(f)\right\|_{L^{q}\left(w^{q / p}\right)} \\
& \quad \leq C \prod_{j=1}^{k}\left(\sum_{\left|\alpha_{j}\right|=m_{j}}\left\|D^{\alpha_{j}} b_{j}\right\|_{\operatorname{Lip}_{\beta}(w)}\right)\|f\|_{L^{p}(w) .}
\end{aligned}
$$

This completes the proof of the theorem.

\section{Applications}

In this section we shall apply Theorems 1 and 2 of the paper to some particular operators such as the Calderón-Zygmund singular integral operator and the fractional integral operator.

Application 1 Calderón-Zygmund singular integral operator. 
Let $T$ be the Calderón-Zygmund operator (see $[17,18])$. The multilinear operator related to $T$ is defined by

$$
T^{b}(f)(x)=\int \frac{R_{m+1}(b ; x, y)}{|x-y|^{m-k}} K(x, y) f(y) d y
$$

Then Theorem 1 holds for $T^{b}$ with $\delta=0$.

Application 2 Fractional integral operator with rough kernel.

For $0<\delta<n$, let $T_{\delta}$ be the fractional integral operator with rough kernel defined by (see $[8,17,18])$

$$
T_{\delta} f(x)=\int_{R^{n}} \frac{\Omega(x-y)}{|x-y|^{n-\delta}} f(y) d y,
$$

which is the fractional integral operator when $\Omega=1$ (see [8]). The multilinear operator related to $T_{\delta}$ is defined by

$$
T_{\delta}^{b} f(x)=\int_{R^{n}} \frac{R_{m+1}(b ; x, y)}{|x-y|^{m+n-k-\delta}} \Omega(x-y) f(y) d y,
$$

where $\Omega$ is homogeneous of degree zero on $R^{n}, \int_{S^{n-1}} \Omega\left(x^{\prime}\right) d \sigma\left(x^{\prime}\right)=0$ and $\Omega \in \operatorname{Lip}_{\varepsilon}\left(S^{n-1}\right)$ for some $0<\varepsilon \leq 1$, that is, there exists a constant $M>0$ such that for any $x, y \in S^{n-1}$, $|\Omega(x)-\Omega(y)| \leq M|x-y|^{\varepsilon}$. Then Theorem 2 holds for $T_{\delta}^{b}$.

\section{Competing interests}

The authors declare that they have no competing interests.

\section{Authors' contributions}

XZ carried out the Theorem 1 studies, participated in the disscussion of Theorem 2. CH is the corresponding author, designed the outline of this paper, carried out the Theorem 1 and Theorem 2 studies, participated in the disscussion of Applications. HH carried out the Theorem 2 studies, participated in the disscussion of Applications. LL participated in the disscussion of Applications and drafted the manuscript. All authors read and approved the final manuscript.

\section{Acknowledgements}

This work was jointly supported by the National Natural Science Foundation of China under Grant No. 11101053, the Key Project of Chinese Ministry of Education under Grant no. 211118, the Scientific Research Funds of Hunan Provincial Science and Technology Department of China no. 2013SK3143, the Hunan Provincial NSF no. 11JJ1001, the Scientific Research Fund of Hunan Provincial Education Department of China (no. 12C0028) and the Open Fund Project of Key Research Institute of Philosophies and Social Sciences in Hunan Universities (no. 12FEFM05).

Received: 27 February 2013 Accepted: 18 May 2013 Published: 1 July 2013

\section{References}

1. Cohen, J: A sharp estimate for a multilinear singular integral on $R^{n}$. Indiana Univ. Math. J. 30, 693-702 (1981)

2. Cohen, J, Gosselin, J: On multilinear singular integral operators on $R^{n}$. Stud. Math. 72, 199-223 (1982)

3. Cohen, J, Gosselin, J: A BMO estimate for multilinear singular integral operators. III. J. Math. 30, 445-465 (1986)

4. Coifman, R, Meyer, Y: Wavelets, Calderón-Zygmund and Multilinear Operators. Cambridge Studies in Advanced Math., vol. 48. Cambridge University Press, Cambridge (1997)

5. Coifman, RR, Rochberg, R, Weiss, G: Factorization theorems for Hardy spaces in several variables. Ann. Math. 103, 611-635 (1976)

6. Pérez, C: Endpoint estimate for commutators of singular integral operators. J. Funct. Anal. 128, 163-185 (1995)

7. Pérez, C, Trujillo-Gonzalez, R: Sharp weighted estimates for multilinear commutators. J. Lond. Math. Soc. 65, 672-692 (2002)

8. Chanillo, S: A note on commutators. Indiana Univ. Math. J. 31, 7-16 (1982)

9. Chen, WG: Besov estimates for a class of multilinear singular integrals. Acta Math. Sin. 16, 613-626 (2000)

10. Ding, Y, Lu, SZ, Yabuta, K: Multilinear singular and fractional integrals. Acta Math. Sin. 22, 347-356 (2006)

11. Janson, S: Mean oscillation and commutators of singular integral operators. Ark. Mat. 16, 263-270 (1978) 
12. Paluszynski, M: Characterization of the Besov spaces via the commutator operator of Coifman, Rochberg and Weiss. Indiana Univ. Math. J. 44, 1-17 (1995)

13. Bloom, S: A commutator theorem and weighted BMO. Trans. Am. Math. Soc. 292, 103-122 (1985)

14. $\mathrm{Hu}, \mathrm{B}, \mathrm{Gu}$, J: Necessary and sufficient conditions for boundedness of some commutators with weighted Lipschitz spaces. J. Math. Anal. Appl. 340, 598-605 (2008)

15. Pérez, C, Pradolini, G: Sharp weighted endpoint estimates for commutators of singular integral operators. Mich. Math. J. 49, 23-37 (2001)

16. Pérez, C, Trujillo-Gonzalez, R: Sharp weighted estimates for vector-valued singular integral operators and commutators. Tohoku Math. J. 55, 109-129 (2003)

17. Garcia-Cuerva, J, Rubio de Francia, JL: Weighted Norm Inequalities and Related Topics. North-Holland Mathematics Studies, vol. 116. North-Holland, Amsterdam (1985)

18. Stein, EM: Harmonic Analysis: Real Variable Methods, Orthogonality and Oscillatory Integrals. Princeton University Press, Princeton (1993)

19. Muckenhoupt, B, Wheeden, RL: Weighted norm inequalities for fractional integral. Trans. Am. Math. Soc. 192, 261-274 (1974)

20. Garcia-Cuerva, J: Weighted $H^{P}$ Spaces. Diss. Math., vol. 162 (1979)

doi:10.1186/1029-242X-2013-303

Cite this article as: Zhou et al.: Inequality estimates for the boundedness of multilinear singular and fractional integral operators. Journal of Inequalities and Applications 2013 2013:303.

\section{Submit your manuscript to a SpringerOpen ${ }^{\circ}$ journal and benefit from:}

- Convenient online submission

Rigorous peer review

- Immediate publication on acceptance

Open access: articles freely available online

- High visibility within the field

- Retaining the copyright to your article 